\title{
Results on Difference Analogues of Valiron-Mohon'ko Theorem
}

\author{
Ran Ran Zhang' and Zhi Bo Huang ${ }^{2}$ \\ ${ }^{1}$ Department of Mathematics, Guangdong University of Education, Guangzhou 510303, China \\ ${ }^{2}$ School of Mathematical Sciences, South China Normal University, Guangzhou 510631, China \\ Correspondence should be addressed to Ran Ran Zhang; zhrr19820315@163.com
}

Received 6 April 2013; Accepted 4 May 2013

Academic Editor: Allan Peterson

Copyright (C) 2013 R. R. Zhang and Z. B. Huang. This is an open access article distributed under the Creative Commons Attribution License, which permits unrestricted use, distribution, and reproduction in any medium, provided the original work is properly cited.

The classical Valiron-Mohon'ko theorem has many applications in the study of complex equations. In this paper, we investigate rational functions in $f(z)$ and the shifts of $f(z)$. We get some results on their characteristic functions. These results may be viewed as difference analogues of Valiron-Mohon'ko theorem.

\section{Introduction and Results}

We use the basic notions of Nevanlinna's theory in this work (see $[1,2])$. Let $f(z)$ be a meromorphic function. We say that a meromorphic function $\alpha(z)$ is a small function of $f(z)$ if $T(r, \alpha)=S(r, f)$, where $S(r, f)=o(T(r, f))$ outside a possible exceptional set of finite logarithmic measure.

The Valiron-Mohon'ko theorem has been proved to be an extremely useful tool in the study of meromorphic solutions of differential, difference, and functional equations. It is stated as follows.

Theorem A (see [3, page 29]). Let $f$ be a meromorphic function. Then for all irreducible rational functions in $f$

$$
R(z, f)=\frac{\sum_{i=0}^{p} a_{i}(z) f^{i}}{\sum_{j=0}^{q} b_{j}(z) f^{j}}
$$

with meromorphic coefficients $a_{i}(z), b_{j}(z)$ such that

$$
\begin{array}{ll}
T\left(r, a_{i}\right)=S(r, f), & i=0, \ldots, p, \\
T\left(r, b_{j}\right)=S(r, f), & j=0, \ldots, q,
\end{array}
$$

the characteristic function of $R(r, f(z))$ satisfies

$$
T(r, R(z, f))=\max \{p, q\} T(r, f)+S(r, f) .
$$

Recently, a number of papers have focused on difference analogues of Nevanlinna's theory; see, for instance, [4-12].
Among these papers, difference polynomials are investigated extensively (see [5, 9-11]). But the difference analogues of Valiron-Mohon'ko theorem have not been established. In this paper, we are devoted to this work.

A difference polynomial of $f(z)$ is an expression of the form

$$
H(z, f)=\sum_{\lambda \in J} a_{\lambda}(z) \prod_{j=1}^{\tau_{\lambda}} f\left(z+\delta_{\lambda, j}\right)^{\mu_{\lambda, j}}
$$

where $J$ is an index set, $\delta_{\lambda, j}$ are complex constants, and $\mu_{\lambda, j}$ are nonnegative integers. In what follows, we assume that the coefficients of difference polynomials are, unless otherwise stated, small functions. The maximal total degree of $H(z, f)$ in $f(z)$ and the shifts of $f(z)$ is defined by

$$
\operatorname{deg}_{f} H=\max _{\lambda \in J} \sum_{j=1}^{\tau_{\lambda}} \mu_{\lambda, j} .
$$

First, we investigate the rational function

$$
R_{1}(z, f)=\frac{P(z, f)}{d_{1}(z) f(z+c)+d_{0}(z)},
$$

where $c$ is an arbitrary complex number, and $d_{0}(z)$ and $d_{1}(z)$ are small functions of $f(z)$ with $d_{0}(z) \neq \equiv$ or $d_{1}(z) \not \equiv 0$. Our result is stated as follows.

Theorem 1. Let $f(z)$ be a meromorphic function of finite order such that $N(r, f)=S(r, f)$. Suppose that $P(z, f) \not \equiv 0$ is a 
difference polynomial in $f(z)$ and that $R_{1}(z, f)$ is of the form (6). Then

$$
T\left(r, R_{1}\right) \leq\left(\operatorname{deg}_{f} P\right) T(r, f)+S(r, f) .
$$

In many papers (see, for instance, [7, 13, 14]), linear difference expressions often appear. Concerning their characteristic functions, we have the following corollary, which is obtained easily from Theorem 1 .

Corollary 2. Let $f(z)$ be a meromorphic function of finite order such that $N(r, f)=S(r, f)$. Suppose that $L(z, f) \neq \equiv$ is a linear combination in $f(z)$ and the shifts of $f(z)$. Then

$$
T(r, L) \leq T(r, f)+S(r, f) .
$$

Next we consider the rational function

$$
R_{2}(z, f)=\frac{P(z, f)}{f\left(z+c_{1}\right) \cdots f\left(z+c_{n}\right)},
$$

where $c_{1}, \ldots, c_{n}$ are different complex constants. We get the following result.

Theorem 3. Let $f(z)$ be a meromorphic function of finite order such that $N(r, f)=S(r, f)$. Suppose that $P(z, f) \not \equiv 0$ is a difference polynomial in $f(z)$ and that $R_{2}(z, f)$ is of the form (9). Then

$$
T\left(r, R_{2}\right) \leq \max \left\{\operatorname{deg}_{f} P, n\right\} T(r, f)+S(r, f) .
$$

As for the general rational function in $f(z)$ and the shifts of $f(z)$,

$$
R_{3}(z, f)=\frac{P(z, f)}{Q(z, f)},
$$

we get the following two results.

Theorem 4. Let $f(z)$ be a meromorphic function of finite order such that $N(r, f)=S(r, f)$. Suppose that $P(z, f) \neq \equiv$ and $Q(z, f) \quad \neq \equiv 0$ are difference polynomials in $f(z)$ and that $R_{3}(z, f)$ is of the form (11).

(i) If $\operatorname{deg}_{f} P \geq \operatorname{deg}_{f} Q$ and $P(z, f)$ contains just one term of maximal total degree, then

$$
T\left(r, R_{3}\right) \geq\left(\operatorname{deg}_{f} P-\operatorname{deg}_{f} Q\right) T(r, f)+S(r, f) .
$$

(ii) If $\operatorname{deg}_{f} P \leq \operatorname{deg}_{f} Q$ and $Q(z, f)$ contains just one term of maximal total degree, then

$$
T\left(r, R_{3}\right) \geq\left(\operatorname{deg}_{f} Q-\operatorname{deg}_{f} P\right) T(r, f)+S(r, f) .
$$

Theorem 5. Let $f(z)$ be a meromorphic function of finite order such that $N(r, f)+N(r, 1 / f)=S(r, f)$. Suppose that $P(z, f) \not \equiv 0$ and $Q(z, f)$ \# 0 are difference polynomials in $f(z)$ and that $R_{3}(z, f)$ is of the form (11). Then

$$
T\left(r, R_{3}\right) \leq \max \left\{\operatorname{deg}_{f} P, \operatorname{deg}_{f} Q\right\} T(r, f)+S(r, f) .
$$

The following two examples show that the results in Theorems 1-5 are sharp; that is, " $\leq$ " and " $\geq$ " cannot be replaced by "<", ">” or “=”.

Example 6. Let $f(z)=e^{z}$ and

$$
\begin{aligned}
P(z, f)= & f(z)^{2} f(z+\pi i)+f(z)^{2} \\
& +2 f(z+\pi i) f(z)+2 f(z)+f(z+\pi i)+1 .
\end{aligned}
$$

Let

$$
R_{11}(z, f)=\frac{P(z, f)}{f(z+\pi i)+2}, \quad R_{12}(z, f)=\frac{P(z, f)}{f(z+\pi i)+1} .
$$

Then $R_{11}(z, f)=\left(1+e^{z}\right)^{2}\left(1-e^{z}\right) /\left(-e^{z}+2\right)$ and $R_{12}(z, f)=$ $\left(1+e^{z}\right)^{2}$. Clearly,

$$
\begin{aligned}
& T\left(r, R_{11}\right)=3 T(r, f)+S(r, f), \\
& T\left(r, R_{12}\right)=2 T(r, f)+S(r, f) .
\end{aligned}
$$

Therefore,

$$
\begin{aligned}
& \left.\quad \operatorname{deg}_{f} P-1\right) T(r, f)+S(r, f) \\
& \quad<T\left(r, R_{11}\right)=\left(\operatorname{deg}_{f} P\right) T(r, f)+S(r, f), \\
& \left(\operatorname{deg}_{f} P-1\right) T(r, f)+S(r, f) \\
& \quad=T\left(r, R_{12}\right)<\left(\operatorname{deg}_{f} P\right) T(r, f)+S(r, f) .
\end{aligned}
$$

Example 7. Let $f(z)=\sin z$ and

$$
P(z, f)=f\left(z+\frac{\pi}{2}\right)^{2} f(z)+f(z)^{2}+f(z+\pi) f(z)-f(z) .
$$

Let

$$
R_{21}(z, f)=\frac{P(z, f)}{f(z+\pi / 2)^{3}}, \quad R_{22}(z, f)=\frac{P(z, f)}{f(z+\pi)^{2}} .
$$

Then $R_{21}(z, f)=-\tan ^{3} z$ and $R_{22}(z, f)=-\sin z$. Clearly,

$$
\begin{aligned}
& T\left(r, R_{21}\right)=3 T(r, f)+S(r, f), \\
& T\left(r, R_{22}\right)=T(r, f)+S(r, f) .
\end{aligned}
$$

Therefore,

$$
\begin{aligned}
& \left(\operatorname{deg}_{f} P-3\right) T(r, f)+S(r, f) \\
& \quad<T\left(r, R_{21}\right)=\left(\operatorname{deg}_{f} P\right) T(r, f)+S(r, f), \\
& \left(\operatorname{deg}_{f} P-2\right) T(r, f)+S(r, f) \\
& \quad=T\left(r, R_{22}\right)<\left(\operatorname{deg}_{f} P\right) T(r, f)+S(r, f) .
\end{aligned}
$$




\section{Proof of Theorem 1}

We need the following lemmas for the proof of Theorem 1.

The difference analogue of the logarithmic derivative lemma was given by Halburd-Korhonen [8, Corollary 2.2] and Chiang-Feng [7, Corollary 2.6], independently. The following Lemma 8 is a variant of [8, Corollary 2.2].

Lemma 8. Let $f(z)$ be a nonconstant meromorphic function of finite order, and let $\eta_{1}, \eta_{2}$ be two arbitrary complex numbers. Then,

$$
m\left(r, \frac{f\left(z+\eta_{1}\right)}{f\left(z+\eta_{2}\right)}\right)=S(r, f) .
$$

In the remark of [15, page 15], it is pointed out that the following lemma holds.

Lemma 9. Let $f(z)$ be a nonconstant finite order meromorphic function and let $c \neq 0$ be an arbitrary complex number. Then,

$$
\begin{aligned}
& T(r+|c|, f)=T(r, f)+S(r, f), \\
& N(r+|c|, f)=N(r, f)+S(r, f) .
\end{aligned}
$$

Let $f(z)$ be a meromorphic function. It is shown in [16, page 66] that for an arbitrary $c \neq 0$, the following inequalities:

$$
\begin{aligned}
(1+o(1)) T(r-|c|, f(z)) & \leq T(r, f(z+c)) \\
& \leq(1+o(1)) T(r+|c|, f(z))
\end{aligned}
$$

hold as $r \rightarrow \infty$. From its proof we see that the above relations are also true for counting functions. So by these relations and Lemma 9, we get the following lemma.

Lemma 10. Let $f(z)$ be a nonconstant finite order meromorphic function and let $c \neq 0$ be an arbitrary complex number. Then,

$$
\begin{aligned}
T(r, f(z+c)) & =T(r, f)+S(r, f), \\
N(r, f(z+c)) & =N(r, f)+S(r, f), \\
N\left(r, \frac{1}{f(z+c)}\right) & =N\left(r, \frac{1}{f}\right)+S(r, f) .
\end{aligned}
$$

Remark 11. In [7], Chiang and Feng proved a similar result. Let $f(z)$ be a meromorphic function with $\sigma(f)<\infty$, and let $\eta \neq 0$ be fixed; then for each $\varepsilon>0$, we have

$$
T(r, f(z+\eta))=T(r, f)+O\left(r^{\sigma(f)-1+\varepsilon}\right)+O(\log r) .
$$

Proof of Theorem 1. Let

$$
P(z, f)=\sum_{\lambda \in I} a_{\lambda}(z) \prod_{j=1}^{\sigma_{\lambda}} f\left(z+\alpha_{\lambda, j}\right)^{l_{\lambda, j}},
$$

and $\operatorname{deg}_{f} P=p$.
Rearranging the expression of $P(z, f)$ by collecting together all terms having the same total degree, we get

$$
P(z, f)=\sum_{i=0}^{p} h_{i}(z) f(z)^{i},
$$

where, for $i=0, \ldots, p$,

$$
\begin{aligned}
h_{i}(z) & =\sum_{\lambda \in I_{i}} a_{\lambda}(z) \prod_{j=1}^{\sigma_{\lambda}}\left(\frac{f\left(z+\alpha_{\lambda, j}\right)}{f(z)}\right)^{l_{\lambda, j}}, \\
I_{i} & =\left\{\lambda \in I \mid \sum_{j=1}^{\sigma_{\lambda}} l_{\lambda, j}=i\right\} .
\end{aligned}
$$

Since the coefficients $a_{\lambda}(z)$ of $P(z, f)$ are small functions of $f(z)$, we have

$$
m\left(r, a_{\lambda}\right) \leq T\left(r, a_{\lambda}\right)=S(r, f) .
$$

So by Lemma 8 , we have, for all $i=0,1, \ldots, p$ the estimates

$$
m\left(r, h_{i}\right)=S(r, f) .
$$

Without loss of generality, we may assume $c=0$ in (6). Otherwise, substituting $z-c$ for $z$, we get

$$
R_{1}(z-c, f)=\frac{P(z-c, f)}{d_{1}(z-c) f(z)+d_{0}(z-c)} .
$$

By Lemma 10, we see that

$$
T\left(r, R_{1}(z-c, f)\right)=T\left(r, R_{1}(z, f)\right)+S(r, f) .
$$

So, in the following discussion, we only discuss the form

$$
R_{1}(z, f)=\frac{P(z, f)}{d_{1}(z) f(z)+d_{0}(z)} .
$$

Assume first that $d_{1}(z)=0$. Clearly, we may assume that $d_{0}(z)=1$. By (29), we get

$$
\begin{aligned}
R_{1}(z, f)= & P(z, f) \\
= & h_{p}(z) f(z)^{p}+h_{p-1}(z) f(z)^{p-1} \\
& +\cdots+h_{1}(z) f(z)+h_{0}(z) .
\end{aligned}
$$

If $p=1$, then $R_{1}(z, f)=h_{1}(z) f(z)+h_{0}(z)$. So by (32), we get

$$
m\left(r, R_{1}\right) \leq m(r, f)+S(r, f) .
$$

If $p>1$, then rewrite $R_{1}(z, f)$ in the form

$$
R_{1}(z, f)=f(z)\left(h_{p}(z) f(z)^{p-1}+\cdots+h_{1}(z)\right)+h_{0}(z) .
$$

So we have

$$
\begin{aligned}
m\left(r, R_{1}\right) \leq & m(r, f) \\
& +m\left(r, h_{p}(z) f(z)^{p-1}+\cdots+h_{1}(z)\right)+S(r, f) .
\end{aligned}
$$


By (39) and the inductive argument, we have

$$
m\left(r, R_{1}\right) \leq p m(r, f)+S(r, f) .
$$

To estimate $N\left(r, R_{1}\right)$, we use the form

$$
R_{1}(z, f)=P(z, f)=\sum_{\lambda \in I} a_{\lambda}(z) \prod_{j=1}^{\sigma_{\lambda}} f\left(z+\alpha_{\lambda, j}\right)^{l_{\lambda, j}} .
$$

Clearly,

$$
\begin{aligned}
& N\left(r, R_{1}\right) \\
& \quad \leq \sum_{\lambda \in I}\left(N\left(r, a_{\lambda}\right)+\sum_{j=1}^{\sigma_{\lambda}} l_{\lambda, j} N\left(r, f\left(z+\alpha_{\lambda, j}\right)\right)\right)+O(1) .
\end{aligned}
$$

So by (31), $N(r, f)=S(r, f)$, and Lemma 10, we get

$$
N\left(r, R_{1}\right)=S(r, f) .
$$

Combining this equality with (40), we get

$$
T\left(r, R_{1}\right) \leq p T(r, f)+S(r, f),
$$

and we have completed the case $d_{1}(z)=0$.

We now proceed to the case $d_{1}(z) \neq 0$. Clearly, in this case we may assume that $d_{1}(z)=1$. By (29), we see that (6) becomes

$$
\begin{aligned}
& R_{1}(z, f) \\
& =\left(h_{p}(z) f(z)^{p}+h_{p-1}(z) f(z)^{p-1}\right. \\
& \left.\quad+\cdots+h_{1}(z) f(z)+h_{0}(z)\right) \\
& \quad \times\left(f(z)+d_{0}(z)\right)^{-1} .
\end{aligned}
$$

By (45), we get

$$
\begin{aligned}
R_{1}(z, f) & \\
= & h_{p}(z) f(z)^{p-1} \\
& +\left(h_{p-1}^{*}(z) f(z)^{p-1}+h_{p-2}(z) f(z)^{p-2}\right. \\
& \left.\quad+\cdots+h_{1}(z) f(z)+h_{0}(z)\right) \\
& \quad \times\left(f(z)+d_{0}(z)\right)^{-1} \\
= & h_{p}(z) f(z)^{p-1}+h_{p-1}^{*}(z) f(z)^{p-2} \\
& +\frac{h_{p-2}^{*}(z) f(z)^{p-2}+\cdots+h_{1}(z) f(z)+h_{0}(z)}{f(z)+d_{0}(z)} \\
= & \ldots \\
= & h_{p}(z) f(z)^{p-1}+h_{p-1}^{*}(z) f(z)^{p-2} \\
& +\cdots+h_{2}^{*}(z) f(z)+h_{1}^{*}(z)+\frac{h_{0}^{*}(z)}{f(z)+d_{0}(z)},
\end{aligned}
$$

where

$$
\begin{gathered}
h_{p-1}^{*}(z)=h_{p-1}(z)-h_{p}(z) d_{0}(z), \\
h_{p-2}^{*}(z)=h_{p-2}(z)-h_{p-1}^{*}(z) d_{0}(z), \\
\vdots \\
h_{1}^{*}(z)=h_{1}(z)-h_{2}^{*}(z) d_{0}(z), \\
h_{0}^{*}(z)=h_{0}(z)-h_{1}^{*}(z) d_{0}(z) .
\end{gathered}
$$

By (32), we get, for $j=0,1, \ldots, p-1$, the estimates

$$
m\left(r, h_{j}^{*}\right)=S(r, f) .
$$

By (46), using the same method as in (36)-(40), we get

$$
\begin{aligned}
& m\left(r, R_{1}\right) \\
& \leq m\left(r, h_{p}(z) f(z)^{p-1}+h_{p-1}^{*}(z) f(z)^{p-2}+\cdots+h_{1}^{*}(z)\right) \\
& \quad+m\left(r, \frac{h_{0}^{*}(z)}{f(z)+d_{0}(z)}\right) \\
& \leq(p-1) m(r, f) \\
& \quad+m\left(r, \frac{1}{f(z)+d_{0}(z)}\right)+S(r, f) .
\end{aligned}
$$

To estimate $N\left(r, R_{1}\right)$, we use the form

$$
\begin{aligned}
R_{1}(z, f) & =\frac{P(z, f)}{f(z)+d_{0}(z)} \\
& =\frac{\sum_{\lambda \in I} a_{\lambda}(z) \prod_{j=1}^{\sigma_{\lambda}} f\left(z+\alpha_{\lambda, j}\right)^{l_{\lambda, j}}}{f(z)+d_{0}(z)} .
\end{aligned}
$$

By $(31), N(r, f)=S(r, f)$, and Lemma 10, we get

$$
N\left(r, R_{1}\right)=N\left(r, \frac{1}{f(z)+d_{0}(z)}\right)+S(r, f) .
$$

Combining this equality with (49), we get

$$
\begin{aligned}
T\left(r, R_{1}\right) \leq & (p-1) m(r, f) \\
& +T\left(r, \frac{1}{f(z)+d_{0}(z)}\right)+S(r, f) \\
\leq & p T(r, f)+S(r, f) .
\end{aligned}
$$

Theorem 1 is proved.

\section{Proof of Theorem 3}

Proof. Let $P(z, f)$ be of the form (28) and $\operatorname{deg}_{f} P=p$. Rearranging the expression of $P(z, f)$, we get (29) and (30). We only discuss the case $p \geq n$ since the case $p<n$ is easier. 
Rewrite $R_{2}(z, f)$ in the form

$$
R_{2}(z, f)=\frac{P(z, f)}{s(z) f(z)^{n}},
$$

where

$$
s(z)=\frac{f\left(z+c_{1}\right) \cdots f\left(z+c_{n}\right)}{f(z)^{n}} .
$$

By Lemma 8, we get

$$
m\left(r, \frac{1}{s}\right)=S(r, f) .
$$

By (29) and (53), we get

$$
\begin{aligned}
R_{2}(z, f)= & \frac{\sum_{i=0}^{p} h_{i}(z) f(z)^{i}}{s(z) f(z)^{n}} \\
= & \sum_{i=n}^{p} \frac{h_{i}(z)}{s(z)} f(z)^{i-n} \\
& +\frac{h_{n-1}(z) f(z)^{n-1}+\cdots+h_{0}(z)}{s(z) f(z)^{n}} \\
= & \sum_{i=n}^{p} \frac{h_{i}(z)}{s(z)} f(z)^{i-n} \\
& +\sum_{j=1}^{n} \frac{h_{n-j}(z)}{s(z)}\left(\frac{1}{f(z)}\right)^{j} .
\end{aligned}
$$

By (32) and (55), we have, for all $i=0, \ldots, p$, the estimates

$$
m\left(r, \frac{h_{i}(z)}{s(z)}\right)=S(r, f) .
$$

By (57), using the same method as in (36)-(40), we get

$$
\begin{aligned}
& m\left(r, \sum_{i=n}^{p} \frac{h_{i}(z)}{s(z)} f(z)^{i-n}\right) \leq(p-n) m(r, f)+S(r, f), \\
& m\left(r, \sum_{j=1}^{n} \frac{h_{n-j}(z)}{s(z)}\left(\frac{1}{f(z)}\right)^{j}\right) \leq n m\left(r, \frac{1}{f}\right)+S(r, f) .
\end{aligned}
$$

Combining the above two inequalities with (56), we get

$$
m\left(r, R_{2}\right) \leq(p-n) m(r, f)+n m\left(r, \frac{1}{f}\right)+S(r, f) .
$$

To estimate $N\left(r, R_{2}\right)$, we use the form

$$
R_{2}(z, f)=\frac{\sum_{\lambda \in I} a_{\lambda}(z) \prod_{j=1}^{\sigma_{\lambda}} f\left(z+\alpha_{\lambda, j}\right)^{l_{\lambda, j}}}{f\left(z+c_{1}\right) \cdots f\left(z+c_{n}\right)} .
$$

By $(31), N(r, f)=S(r, f)$, and Lemma 10, we get

$$
\begin{aligned}
N\left(r, R_{2}\right)= & N\left(r, \frac{1}{f\left(z+c_{1}\right) \cdots f\left(z+c_{n}\right)}\right) \\
& +S(r, f) \leq n N\left(r, \frac{1}{f}\right)+S(\mathrm{r}, f) .
\end{aligned}
$$

Combining this inequality with (59), we get

$$
\begin{aligned}
T\left(r, R_{2}\right) \leq & (p-n) m(r, f)+n T\left(r, \frac{1}{f}\right) \\
& +S(r, f) \leq p T(r, f)+S(r, f) .
\end{aligned}
$$

Theorem 3 is proved.

\section{Proof of Theorem 4}

We need the following lemma for the proof of Theorem 4 .

Lemma 12 (see [11]). Let $f(z)$ be a meromorphic function of finite order such that $N(r, f)=S(r, f)$. Suppose that $H(z, f)$ is a difference polynomial in $f(z)$ and $H(z, f)$ contains just one term of maximal total degree. Then,

$$
T(r, H)=\left(\operatorname{deg}_{f} H\right) T(r, f)+S(r, f) .
$$

Proof of Theorem 4. We have the following.

Case 1. Suppose that $\operatorname{deg}_{f} P \geq \operatorname{deg}_{f} Q$ and $P(z, f)$ contains just one term of maximal total degree.

Let $\operatorname{deg}_{f} P=p$ and $\operatorname{deg}_{f} Q=q$. By Lemma 12, we get

$$
T(r, P)=p T(r, f)+S(r, f) .
$$

By Theorem 1, we get

$$
T(r, Q) \leq q T(r, f)+S(r, f) .
$$

By (11), we get

$$
P(z, f)=R_{3}(z, f) Q(z, f) .
$$

By (64)-(66), we get

$$
\begin{aligned}
p T(r, f)+S(r, f) \\
\quad=T(r, P(z, f)) \\
\quad=T\left(r, R_{3}(z, f) Q(z, f)\right) \\
\quad \leq T\left(r, R_{3}(z, f)\right)+T(r, Q(z, f)) \\
\quad \leq T\left(r, R_{3}(z, f)\right)+q T(r, f)+S(r, f) .
\end{aligned}
$$

So we have,

$$
T\left(r, R_{3}\right) \geq(p-q) T(r, f)+S(r, f) .
$$

Case 2. Suppose that $\operatorname{deg}_{f} P \leq \operatorname{deg}_{f} Q$ and $Q(z, f)$ contains just one term of maximal total degree.

In this case, we consider $1 / R_{3}(z, f)$. Using the same method as in Case 1 , we can easily get

$$
T\left(r, R_{3}\right)=T\left(r, \frac{1}{R_{3}}\right) \geq(q-p) T(r, f)+S(r, f) .
$$

Theorem 4 is proved. 


\section{Proof of Theorem 5}

Proof. Let $P(z, f)$ be of the form (28) and $\operatorname{deg}_{f} P=p$. Let

$$
Q(z, f)=\sum_{\mu \in J} b_{\mu}(z) \prod_{j=1}^{\tau_{\mu}} f\left(z+\beta_{\mu, j}\right)^{m_{\mu, j}}
$$

and $\operatorname{deg}_{f} Q=q$.

Rearranging the expression of $P(z, f)$, we get (29) and (30).

Similarly, rearranging the expression of $Q(z, f)$, we get

$$
Q(z, f)=\sum_{k=0}^{q} t_{k}(z) f(z)^{k}
$$

where, for $k=0, \ldots, q$,

$$
\begin{aligned}
t_{k}(z) & =\sum_{\mu \in J_{k}} b_{\mu}(z) \prod_{j=1}^{\tau_{\mu}}\left(\frac{f\left(z+\beta_{\mu, j}\right)}{f(z)}\right)^{m_{\mu, j},}, \\
J_{k} & =\left\{\lambda \in J \mid \sum_{j=1}^{\tau_{\mu}} m_{\mu, j}=k\right\} .
\end{aligned}
$$

By (29) and (71), we get

$$
R_{3}(z, f)=\frac{\sum_{i=0}^{p} h_{i}(z) f(z)^{i}}{\sum_{k=0}^{q} t_{k}(z) f(z)^{k}} .
$$

Since $N(r, f)+N(r, 1 / f)=S(r, f)$, by Lemma 10, we have, for an arbitrary $\eta$,

$$
\begin{aligned}
N\left(r, \frac{f(z+\eta)}{f(z)}\right) & \leq N\left(r, \frac{1}{f}\right)+N(r, f(z+\eta)) \\
& =N\left(r, \frac{1}{f}\right)+N(r, f)+S(r, f) \\
& =S(r, f) .
\end{aligned}
$$

By (74) and Lemma 8, we have, for an arbitrary $\eta$,

$$
T\left(r, \frac{f(z+\eta)}{f(z)}\right)=S(r, f) .
$$

Since the coefficients $a_{\lambda}(z)$ and $b_{\mu}(z)$ of $P(z, f)$ and $Q(z, f)$ are small functions of $f(z)$, by (30), (72), and (75), we get

$$
\begin{array}{ll}
T\left(r, h_{i}\right)=S(r, f), & i=0, \ldots, p \\
T\left(r, t_{k}\right) & =S(r, f), \quad k=0, \ldots, q .
\end{array}
$$

By (73), we are not clear whether $R_{3}(z, f)$ is an irreducible rational function in $f(z)$. So by Theorem $\mathrm{A}$, we get

$$
T\left(r, R_{3}\right) \leq \max \{p, q\} T(r, f)+S(r, f) .
$$

\section{Acknowledgments}

This work was supported by the National Natural Science Foundation of China (11226090 and 11171119) and Guangdong Natural Science Foundation (S2012040006865).

\section{References}

[1] W. K. Hayman, Meromorphic Functions, Clarendon Press, Oxford, UK, 1964.

[2] L. Yang, Value Distribution Theory and New Research, Science Press, Beijing, China, 1982.

[3] I. Laine, Nevanlinna theory and complex differential equations, Walter de Gruyter, Berlin, Germany, 1993.

[4] W. Bergweiler and J. K. Langley, "Zeros of differences of meromorphic functions," Mathematical Proceedings of the Cambridge Philosophical Society, vol. 142, no. 1, pp. 133-147, 2007.

[5] Z. X. Chen, Z. B. Huang, and X. M. Zheng, "On properties of difference polynomials," Acta Mathematica Scientia B, vol. 31, no. 2, pp. 627-633, 2011.

[6] Z. X. Chen and K. H. Shon, "Estimates for the zeros of differences of meromorphic functions," Science in China A, vol. 52, no. 11, pp. 2447-2458, 2009.

[7] Y.-M. Chiang and S.-J. Feng, "On the nevanlinna characteristic of $f(z+\eta)$ and difference equations in the complex plane," Ramanujan Journal, vol. 16, no. 1, pp. 105-129, 2008.

[8] R. G. Halburd and R. J. Korhonen, "Difference analogue of the lemma on the logarithmic derivative with applications to difference equations," Journal of Mathematical Analysis and Applications, vol. 314, no. 2, pp. 477-487, 2006.

[9] I. Laine and C.-C. Yang, "Clunie theorems for difference and $q$-difference polynomials," Journal of the London Mathematical Society, vol. 76, no. 3, pp. 556-566, 2007.

[10] I. Laine and C.-C. Yang, "Value distribution of difference polynomials," Japan Academy A, vol. 83, no. 8, pp. 148-151, 2007.

[11] R. R. Zhang and Z. X. Chen, "Value distribution of difference polynomials of meromorphic functions," Science China Mathematics, vol. 42, no. 11, pp. 1115-1130, 2012.

[12] R. R. Zhang and Z. X. Chen, "Value distribution of meromorphic functions and their differences," Turkish Journal of Mathematics, vol. 36, no. 3, pp. 395-406, 2012.

[13] R. Korhonen and O. Ronkainen, "Order reduction method for linear difference equations," Proceedings of the American Mathematical Society, vol. 139, no. 9, pp. 3219-3229, 2011.

[14] C.-C. Yang and I. Laine, "On analogies between nonlinear difference and differential equations," Japan Academy A, vol. 86, no. 1, pp. 10-14, 2010.

[15] R. G. Halburd and R. J. Korhonen, "Meromorphic solutions of difference equations, integrability and the discrete Painlevé equations," Journal of Physics A, vol. 40, no. 6, pp. R1-R38, 2007.

[16] A. A. Gol'dberg and I. V. Ostrovskii, Distribution of Values of Meromorphic Functions, Nauka, Moscow, Russia, 1970.

Theorem 5 is proved. 


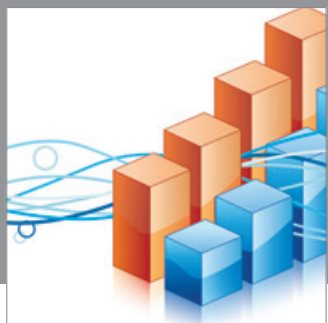

Advances in

Operations Research

mansans

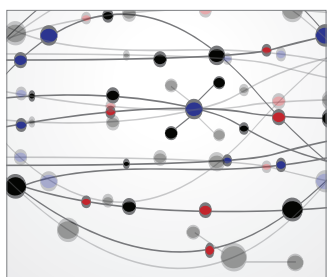

The Scientific World Journal
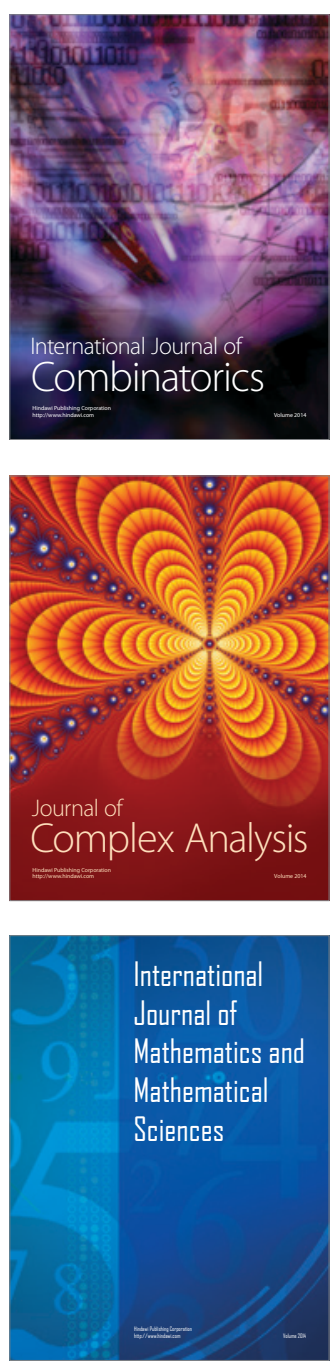
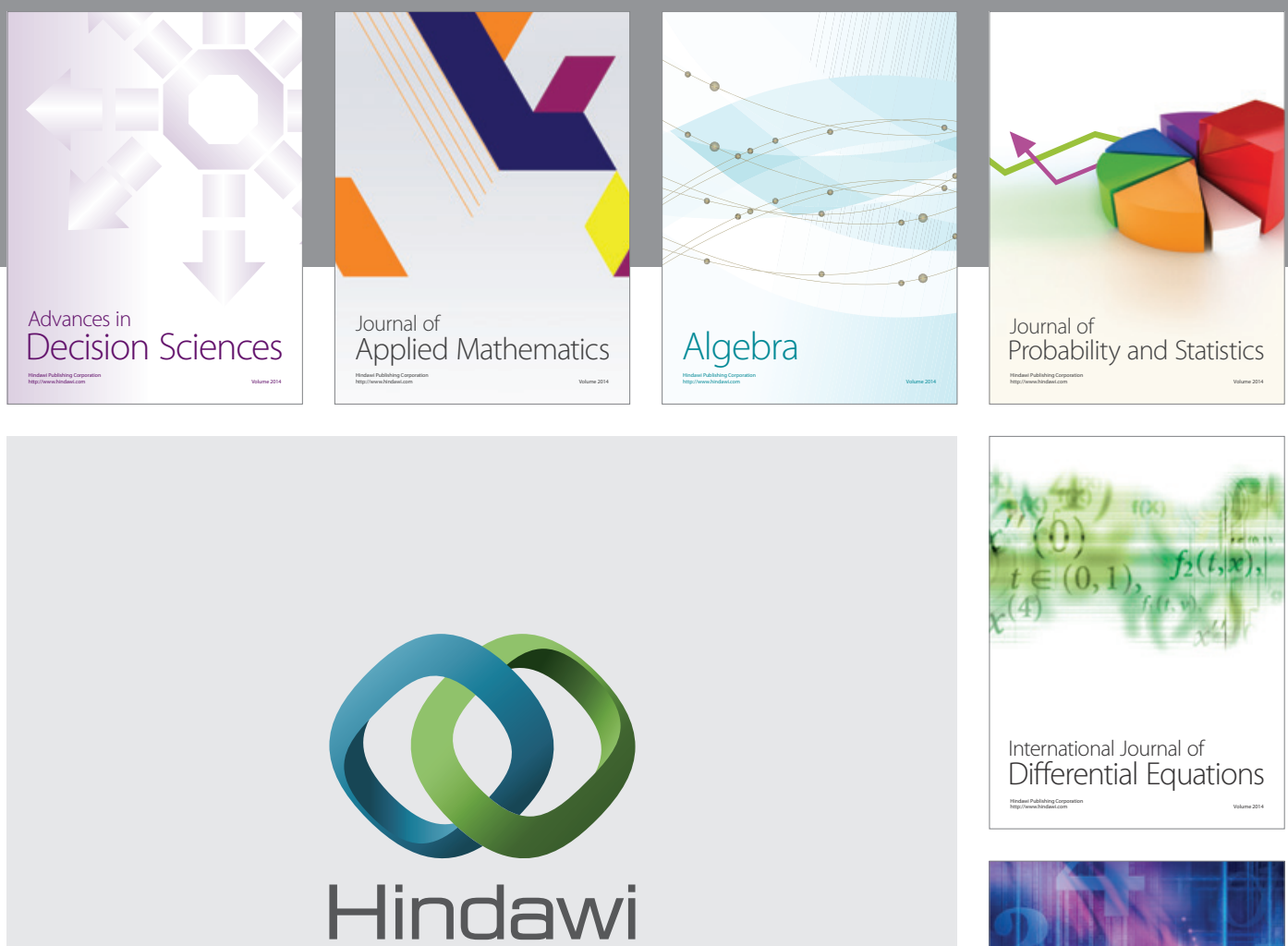

Submit your manuscripts at http://www.hindawi.com
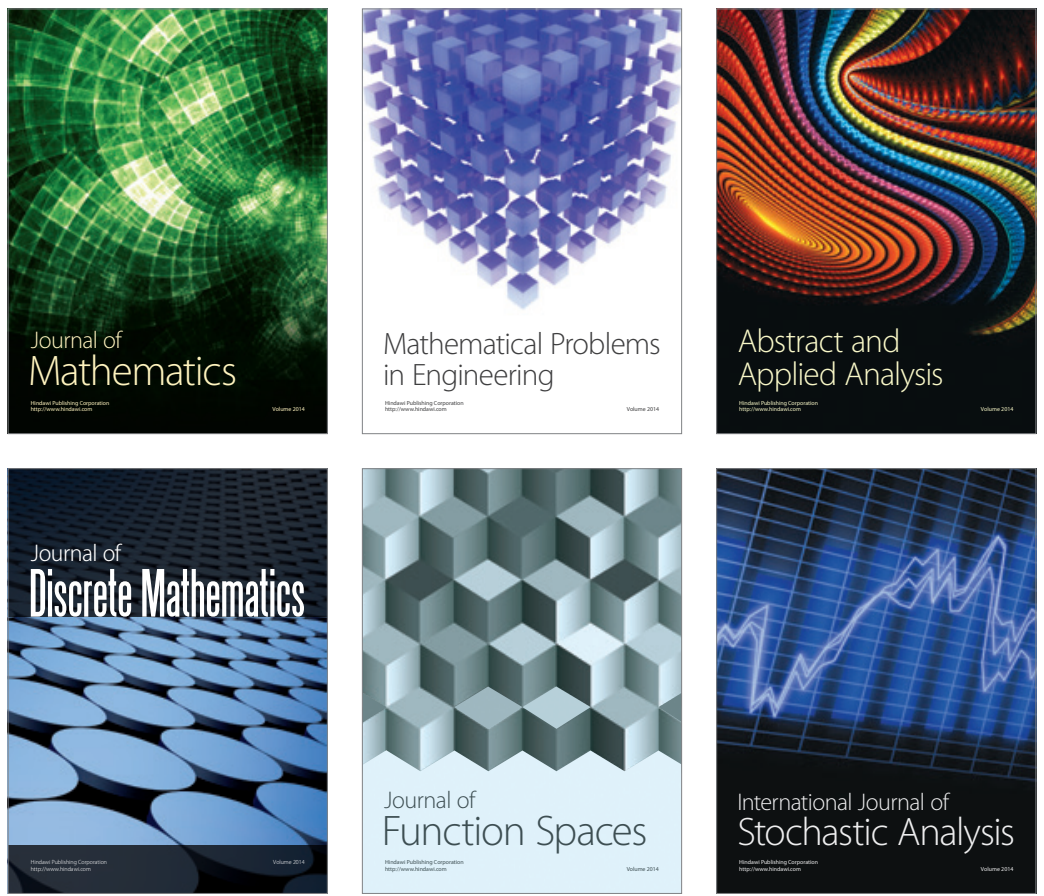

Journal of

Function Spaces

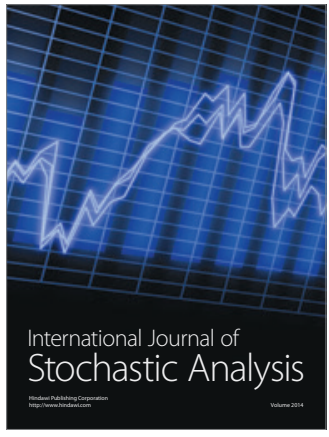

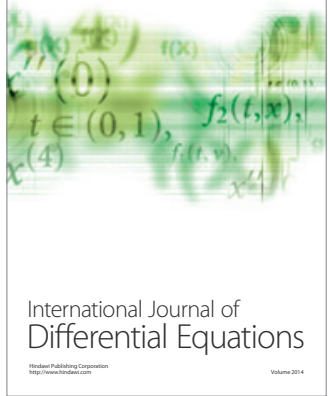
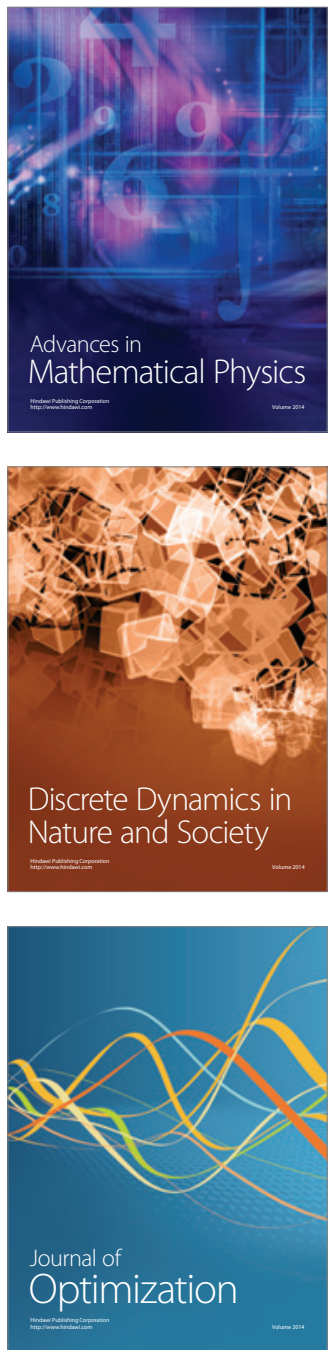